\title{
Towards a Numerical Model of Picosecond Laser-Material Interaction in Bulk Sapphire
}

\author{
L. Capuano, D. de Zeeuw and G.R.B.E. Römer \\ Chair of Laser Processing, Department of Mechanics of Solids, Surfaces \& Systems $\left(M S^{3}\right)$ \\ Faculty of Engineering Technology, University of Twente \\ P.O. Box 217, 7500 AE, Enschede, The Netherlands \\ E-mail: l.capuano@utwente.nl
}

\begin{abstract}
Crystalline sapphire $\left(\mathrm{Al}_{2} \mathrm{O}_{3}\right)$ is a hard and transparent material widely used in industry. When applying IR laser wavelengths, sapphire can be laser-processed inside the bulk (sub-surface) to produce 3D structures, which can find uses, for example, in the production of microfluidic devices. Ultrashort and tightly focused laser pulses trigger several energy absorption mechanisms inside the bulk. The absorbed energy locally modifies the structure of sapphire. Existing (numerical) models of sapphire laser processing describe mainly femtosecond pulsed laser-material interaction (most of them only addressing surface processing) and, in addition, these models do not simulate the laser-induced temperatures of the lattice. Therefore, this study is aimed at a 2D-axisymmetric, time dependent, numerical model of the physics in picosecond laser-material interaction with sapphire. The physical phenomena in model include, but are not limited to: multiphoton absorption, tunneling ionization, avalanche ionization, recombination of carriers, diffusion of carriers and heat diffusion. Based on these phenomena, three quantities are calculated, namely: electron density, electron temperature and lattice temperature. The model was implemented in COMSOL Multiphysics ${ }^{\circledR}$. It was found that, sapphire is modified by the laser radiation only if avalanche ionisation is triggered in the bulk.
\end{abstract}

DOI: 10.2961/jlmn.2018.03.0005

Keywords: sapphire, laser, dielectrics, modeling, sub-surface, processing

\section{Introduction}

Sapphire, (or $\alpha-\mathrm{Al}_{2} \mathrm{O}_{3}$ ), is an aluminium oxide with a crystalline structure, that can be produced synthetically in a variety of shapes and sizes. Sapphire is one of the hardest materials with a hardness ranging from 1835 HV1 to 2039 HV1 [1]. In its crystalline form it is highly chemically inert [1-3]. Because of its wide bandgap [1, 4], sapphire is a good electric insulator and is optically transparent to a broad range of wavelengths going from deep UV to mid infrared in the electromagnetic spectrum [1]. The combination of unique physical and chemical properties and the possibility of producing the material synthetically make sapphire suitable for numerous applications. This includes bearings, smart-phone screens, optics, biomedical applications and microfluidic devices $[2,3,5,6]$.

In order to increase the understanding of the fundamental processes that contribute during subsurface processing of sapphire by means of picosecond pulsed laser sources, a numerical model, which allows simulation of the process under different conditions, is needed. The model should at least be able to describe the electron densities and lattice temperatures that are induced by the absorbed laser energy. This would allow the study of resulting structures and the physics behind the process itself, which will therefore contribute to the fundamental knowledge on subsurface micro-processing of sapphire. In literature, most models address mainly the surface damage induced by ultra-short pulsed lasers [7-12]. These studies typically cover laser pulse durations of less than one picosecond and mostly consider semiconductors, instead of large bandgap dielectric materials, such as sapphire.

In addition, no models are reported, which are suitable for the application to sapphire, allowing to calculate the temperatures in the material as a result of the laser pulse. The literature on modelling of subsurface laser-material interaction that do take into account optical effects, such as focussing and spherical aberrations of a laser beam in sapphire, is quite limited. This is likely due to the complicated electromagnetic description that is required to accurately describe the envelope (caustic) of the laser beam, while taking into account absorption and the challenges that it presents for the numerical simulations.

Models that describe the individual physical phenomena or a part of the laser-material interaction are more frequently reported [13-15]. Although, these models are dominantly applied to ultra-short pulse processing and in studies that show the modelling of electron densities generated, in order to describe or predict the damage threshold for ablation. Therefore, most of the studies reviewed do not take into account electron temperatures.

Based on literature, the governing equations, which make up the model, were selected (see section 2). The equations are implemented in the numerical software tool COMSOL Multiphysics ${ }^{\circledR}$ (see section 3 ). The three main quantities which are calculated are the free electron density, the electron temperature and the lattice temperature (see section 4).

The tables 1, 2 and 3 list the variables, quantities and constants used in this paper. The reader is invited to refer to 
JLMN-Journal of Laser Micro/Nanoengineering Vol. 13, No. 3, 2018

these tables when the variables, quantities and constants are not directly explained in the text.

Table 1 Uppercase variables, quantities and constants

\begin{tabular}{ccc}
\hline Symbol & Description & $\mathrm{Unit}$ \\
\hline$C_{e}$ & $\begin{array}{c}\text { Specific heat capacity of the } \\
\text { electron subsystem }\end{array}$ & $\mathrm{kJ} / \mathrm{kg}$ \\
$C_{l}$ & Specific heat \\
& capacity lattice & $\mathrm{kJ} / \mathrm{kg}$ \\
$E$ & Electric field strength & $\mathrm{V} / \mathrm{m}$ \\
$E_{e}$ & Energy electron subsystem & $\mathrm{eV}$ \\
$E_{g}$ & Band gap energy & $\mathrm{eV}$ \\
$E_{p}$ & Pulse energy \\
$I$ & Optical density & $\mathrm{J}$ \\
$J$ & Current $/ \mathrm{m}^{2}$ \\
$L$ & Loss terms & $\mathrm{A}$ \\
$P$ & Power & $\mathrm{W} / \mathrm{m}^{3}$ \\
$Q$ & Thermal source & $\mathrm{W}$ \\
$S$ & Source term & $\mathrm{W} / \mathrm{m}^{3}$ \\
$T_{e}$ & Temperature electron sub- & $\mathrm{K} \mathrm{or}$ \\
$T_{l}$ & system & $\mathrm{eV}$ \\
$W_{\text {source }}$ & Electron ionization rate & $1 /\left(\mathrm{m}^{3} \mathrm{~s}\right)$ \\
$W_{\text {loss }}$ & Electron reduction rate & $1 /\left(\mathrm{m}^{3} \mathrm{~s}\right)$ \\
\hline & &
\end{tabular}

Table 2 Lowercase variables, quantities and constants

\begin{tabular}{|c|c|c|}
\hline Symbol & Description & Unit \\
\hline$c_{0}$ & Speed of light in vacuum & $\mathrm{m} / \mathrm{s}$ \\
\hline$e$ & Electron charge & $\mathrm{C}$ \\
\hline$\hbar$ & Planck constant & Js \\
\hline$k$ & Wavenumber & $1 / \mathrm{m}$ \\
\hline$k_{0}$ & Reference wavenumber & $1 / \mathrm{m}$ \\
\hline$k_{b}$ & Boltzmann constant & $\mathrm{W} / \mathrm{mK}$ \\
\hline$k_{e}$ & Electron thermal conductivity & $\mathrm{m}^{2} \mathrm{~K} / \mathrm{s}^{2} \mathrm{k}$ \\
\hline$k_{l}$ & Thermal conductivity & $\mathrm{W} / \mathrm{mK}$ \\
\hline$m_{e}$ & Electron mass & $\mathrm{kg}$ \\
\hline$m_{e}^{*}$ & Effective electron mass & $\mathrm{kg}$ \\
\hline$m_{h}^{*}$ & Effective hole mass & $\mathrm{kg}$ \\
\hline$n$ & Refractive index & 1 \\
\hline$n_{2}$ & Nonlinear refractive index & $\mathrm{m}^{2} / \mathrm{W}$ \\
\hline$n_{c r}$ & Critical electron density & 1 \\
\hline$n_{e}$ & Free electron density & $\mathrm{m}^{-3}$ \\
\hline$n_{p h}$ & Number of photons & 1 \\
\hline$n_{v b}$ & Valence band electron density & $\mathrm{m}^{-3}$ \\
\hline$t$ & Time & ps or $\mathrm{s}$ \\
\hline$t_{p}$ & Pulse time & $\mathrm{s}$ \\
\hline$w_{0}$ & Beam radius in focus & $\mathrm{m}$ \\
\hline
\end{tabular}

Table 3 Greek symbols, variables, quantities and constants

\begin{tabular}{|c|c|c|}
\hline Symbol & Description & Unit \\
\hline$\Gamma$ & $\begin{array}{c}\text { Electron phonon coupling } \\
\text { coefficient }\end{array}$ & $\mathrm{J} /(\mathrm{kgKs})$ \\
\hline$\gamma$ & Keyldish parameter & 1 \\
\hline$\delta$ & Impact ionization coefficient & - \\
\hline$\varepsilon_{O}$ & Vacuum permittivity & $\mathrm{F} / \mathrm{m}$ \\
\hline$\eta$ & Electrical impedance & $\Omega$ \\
\hline$\lambda$ & Wavelength & $\mathrm{m}$ \\
\hline$\mu_{e}$ & Electron mobility & $\mathrm{m}^{2} / \mathrm{Vs}$ \\
\hline$\mu_{h}$ & Hole mobility & $\mathrm{m}^{2} / \mathrm{Vs}$ \\
\hline$v_{e}$ & Electron collision frequency & $1 / \mathrm{s}$ \\
\hline$\rho$ & Density & $\mathrm{g} / \mathrm{cm}$ \\
\hline$o$ & Electrical conductivity & $\mathrm{S} / \mathrm{m}$ \\
\hline$\sigma_{a b}$ & Absorption cross-section & $\mathrm{m}$ \\
\hline$\sigma_{C}$ & Ultimate compressive strength & $\mathrm{N} / \mathrm{m}^{2}$ \\
\hline$\sigma_{n}$ & N-photon absorption coeffi- & $\mathrm{m}^{2}$ \\
\hline$\sigma_{t}$ & Ultimate tensile strength & $\mathrm{N} / \mathrm{m}^{2}$ \\
\hline$\tau_{C}$ & $\begin{array}{c}\text { Mean electron-electron colli- } \\
\text { sion time }\end{array}$ & $\mathrm{s}$ \\
\hline$\tau_{e}$ & $\begin{array}{c}\text { Mean electron-hole recombina- } \\
\text { tion time }\end{array}$ & s \\
\hline$\tau_{r}$ & $\begin{array}{c}\text { Characteristic electron lattice } \\
\text { relaxation time }\end{array}$ & $\mathrm{s}$ \\
\hline$\chi$ & Electric susceptibility & 1 \\
\hline$\omega$ & Frequency light & $\mathrm{rad} / \mathrm{s}$ \\
\hline$\omega_{p}$ & Electron-plasma frequency & $\mathrm{rad} / \mathrm{s}$ \\
\hline
\end{tabular}

\section{Model description and governing equations}

Fig. 1 shows a diagram of the structure of the model. The coloured arrows indicate the dependencies of the free electron density, the free electron temperature, the lattice temperature and the beam propagation on the main physical phenomena (blocks in the graph) occurring during and after the laser pulse. There are four "subsystems" that are considered. The first subsystem is the laser intensity distribution (red arrows). Depending on the level of the laser intensity, multiphoton ionization, avalanche ionization, free electron absorption and tunnelling ionization might be triggered. In turn, the laser intensity is reduced by multiphoton ionization and free electron absorption (dashed red arrows). The free electron density subsystem is indicated by the green arrows.

Phenomena that generate free carriers are avalanche ionization, multiphoton ionization and tunnelling ionization. The free electron density will be reduced by recombination effects as free carrier diffusion can be neglected as discussed later in section 2.1. The free electron temperature depends on all phenomena that either affect the free carrier density or lead to a change of the electron kinetic energy. Energy transfer to the lattice, indicated by the black arrows, is described by the well-known two-temperature model [16]. In summary, the model consists of four partial differential equations 
(PDEs) describing: (i) free electron density, (ii) free electron temperature, (iii) lattice temperature, and (iv) beam propagation. These four PDEs are described in the following subsections.

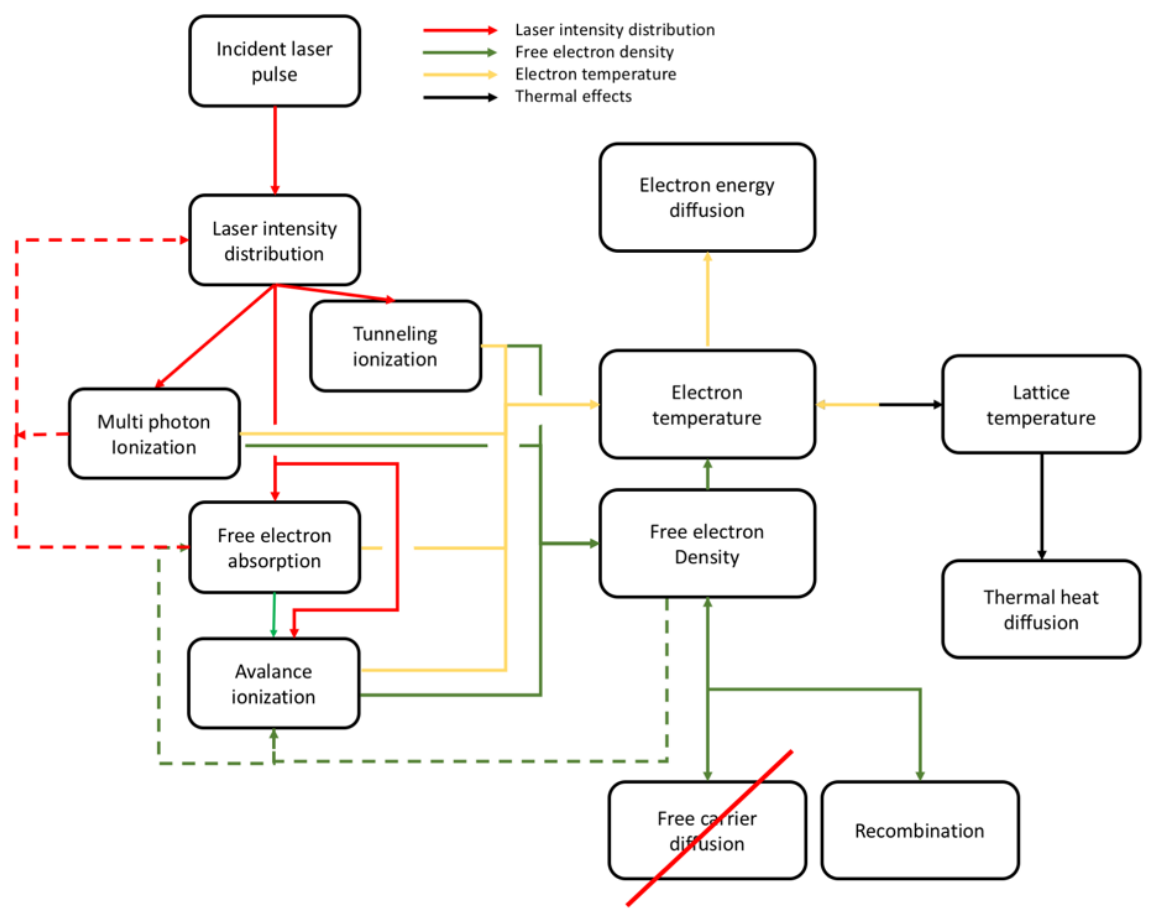

Fig. 1 Diagram of the model, showing the four "subsystems" (arrows) and their dependencies on physical phenomena (blocks).

\subsection{Free electron density}

The free electron density $n_{e}$ can be described by a commonly used electron conservation equation [10-12, 14, 15, 17, 18]:

$\frac{\partial n_{e}(t)}{\partial t}+\frac{1}{e} \frac{\partial J}{\partial x}=W_{\text {source }}+W_{\text {loss: }}$

where $J$ denotes the current and $x$ is the propagation coordinate. The first term on the left hand side of equation (2.1) describes the change of electron density in time. The second term describes the diffusion of electrons in the material. The terms on the right hand side are source and loss terms. The carrier excitation rate source term, $W_{\text {source }}$ in equation (2.1), can be described considering single or multi-photon ionization, tunnelling and avalanche ionization rates. The general expression for the single or multiphoton ionization rate equals

$W_{m p i}(I)=\sigma_{n} I^{n}$

where $n$ denotes the single or multiphoton absorption coefficient. The subscript $n$ indicates the number of photons required to excite a single electron from the valence to the conduction band. Single photon absorption-i.e. $n=1$, which is a linear process, is significant when the photon energy of a photon is equal or larger than the band gap of the material. Multiphoton absorption on the other hand, is an $n$-th order process that will only be significant for sufficiently high laser intensities [19]. Photon ionization in general is only significant at low free-electron densities and when the intensity of the electric field due to the laser fluence is not sufficiently high such that tunneling ionization would dominate [20, 21]. For the wavelength of $1030 \mathrm{~nm}$, which was selected for this study, the photon energy is too low for linear, single photon absorption in sapphire.

The single or multiphoton absorption coefficient $\left(\sigma_{n}\right)$ is a phenomenologically determined parameter that depends on material parameters and processing conditions [12, 22].

In large bandgap dielectrics, the density of thermally excited electrons is close to zero [23]. For short and ultrashort pulse durations, when processing large bandgap dielectrics, single and multiphoton ionization will therefore generate the required electron density to allow significant free electron absorption and subsequently impact ionization to take place [24].

A well-studied approach to model avalanche ionization is the Drude model. The parameters of the Drude model are all known and Jing et al. [24] have shown that the model performs well on fused silica for a laser with $800 \mathrm{~nm}$ wavelength and a pulse duration of $0.3 \mathrm{ps}$. That is, the avalanche ionization rate $W_{a v}$, can be calculated from 
$W_{a v}=\frac{\sigma_{\text {Drude }}}{E_{q}} I(t)$

in which

$\sigma_{\text {Drude }}=\frac{e^{2}}{c \epsilon_{0} n m_{e}^{*}} \frac{\tau_{c}}{1+\omega^{2} \tau_{c}^{2}}$.

The mean electron collision time $\tau_{\mathrm{c}}$ in equation (2.5) is crucial for estimating the avalanche ionization rate and is highly dependent on the material and process conditions such as the electron density, wavelength and effective electron mass. The mean electron collision time $\tau_{\mathrm{c}}$ can be determined phenomenologically or by the expression as developed by Starke et al. [25], which reads

$\tau_{c}=\frac{16 \pi \epsilon_{0}^{2} \sqrt{m_{e}^{*}\left(0.1 E_{g}\right)^{3}}}{\sqrt{2} e^{4} n_{e}(t)}$

This expression increases the applicability of the Drude model under various conditions and when data on the mean electron collision time for various process conditions is limited.

When the absorption of photons is sufficiently high (see section 4), the increased absorption generates a significant amount of free carriers to trigger avalanche ionization [19].

In addition to multiphoton and avalanche ionization, tunneling ionization may occur if the laser intensities are sufficiently high. Multiphoton and tunneling ionization can be described by the same theoretical framework via the socalled Keldysh ionization rate $W_{\mathrm{pi}}$, which is calculated as [20]

$W_{p i}=\frac{2 \omega}{9 \pi}\left(\frac{\omega m_{e}^{*}}{\sqrt{\gamma 1} \hbar^{2}}\right)^{3 / 2} Q(\gamma, \boldsymbol{x}) \times$

$$
\exp \left\{-\pi\langle\boldsymbol{x}+1\rangle \times \frac{\boldsymbol{K}\left(\gamma_{1}\right)-\boldsymbol{E}\left(\gamma_{1}\right)}{\boldsymbol{E}\left(\gamma_{2}\right)}\right\}
$$

and

$$
\begin{array}{r}
Q(\gamma, \boldsymbol{x})=\sqrt{\frac{\pi}{2 \boldsymbol{K}\left(\gamma_{2}\right)}} \times \sum_{i=0}^{\infty} \exp \left\{-i \pi \frac{\boldsymbol{K}\left(\gamma_{1}\right)+\boldsymbol{E}\left(\gamma_{1}\right)}{\boldsymbol{K}\left(\gamma_{2}\right)}\right\} \times \quad(2.8) \\
\boldsymbol{\Phi}\left(\frac{\pi}{2} \sqrt{\frac{(2\langle\boldsymbol{x}+1\rangle-2 \boldsymbol{x}+i)}{\boldsymbol{K}\left(\gamma_{2}\right) \boldsymbol{E}\left(\gamma_{2}\right)}}\right)
\end{array}
$$

and

$\Phi(z)=\int_{0}^{z} \exp \left(y^{2}-z^{2}\right) d y$

and

$\boldsymbol{x}=\frac{\pi}{2} \frac{E_{g}}{\hbar \omega} \frac{\sqrt{1+\gamma^{2}}}{\gamma} \boldsymbol{E}\left(\gamma_{2}\right)$ $\gamma=\frac{\omega}{e} \sqrt{\frac{m_{e}^{*} c n \epsilon_{0} E_{g}}{2 I}}$

Here, the functions $K(\gamma)$ and $E(\gamma)$ represent the complete elliptic integral of the first and second kind respectively. The "crossover" from multiphoton dominated ionization to tunnelling dominated ionization is characterized by the Keldysh parameter, $\gamma$. That is, for $\gamma<<1$, tunnelling ionization dominates and for $\gamma \gg 1$ multiphoton ionization dominates.

The multiphoton ionization rate coincides with the Keldysh photo-ionization rate only for low laser intensity levels. It should be noted that, the validity of the multiphoton ionization rate, when considering a large range of laser intensities, when avalanche ionization is triggered, is questionable.

When electron-hole pairs recombine the electron density is reduced. In sapphire radiative recombination is not significant due to a large radiative recombination lifetime of about 100 ps, when compared to the pulse duration [26]. Therefore, radiative recombination is neglected in the model.

In the case that the pulse duration is shorter than the recombination time $\tau_{\mathrm{e}}$, the recombination of electron-hole pairs is often neglected [7, 8, 27]. However, Auger recombination is included in the model. Typically, the Auger recombination rate $W_{\text {rec }}$ is calculated as $[13,14,16,28-30]$

$W_{\text {rec }}=-\frac{n_{e}(t)}{\tau_{e}}$

in which $\tau_{\mathrm{e}}$ denotes the mean recombination time. In the case of pulse durations equal or longer than the recombination time, or when the decay and behaviour of the electron density, as well as the electron temperature is an important subject of study, recombination needs to be taken into account. For sapphire, the electron-hole recombination time of $\tau_{\mathrm{e}}=1$ ps was applied by Bulgakova et al. [14, 31]. As this recombination time $\tau_{\mathrm{e}}$ is smaller than the pulse duration considered in this study, Auger recombination will be significant.

Besides recombination effects, diffusion of electrons into the substrate also reduce the local electron density. However, for large bandgap dielectrics, the diffusion of free electrons is small due to a high electron-hole mass. Hence, in the case of sapphire, electron diffusion can be neglected [4, 22] (see also Fig. 1).

For the set of (simulation) parameters considered, the Keyldish parameter is never exclusively $\gamma>>1$ or $\gamma<<1$. Hence, the model will only be realistic when both multiphoton and tunnelling ionization are considered. Hence, the full Keldysh ionization rate $S_{m p i}$ is included in the model. Substituting the relevant expressions into equation (2.1), and neglecting carrier diffusion, will result in the PDE describing the conservation of electron-hole pairs, which will be used to describe the carrier density in the numerical model as

$\frac{\partial n_{e}(t)}{\partial t}=W_{p i}+W_{a v, \text { Drude }} n_{e}(t)-W_{\text {rec }}$

and 


\subsection{Free electron temperature}

The absorbed laser energy will first be "stored in" the free electrons, before it is transferred to the lattice via electron-phonon scattering [16]. The energy (density) in the free electron subsystem is the sum of the kinetic energy and band gap energy per unit of volume [21]. The kinetic energy distribution can take several "shapes". That is, when high carrier densities are encountered $\left(n_{e} \approx 10^{17}-10^{18} \mathrm{~W} / \mathrm{cm}^{3}\right)$, the carrier-carrier interactions will force the shape of the distribution into a Maxwell-Boltzmann (also referred to as Maxwellian) distribution [32, 33]. For a Maxwellian distribution the energy density of the electron-hole pairs can be computed from [32]

$U=n_{e}\left(E_{g}+\frac{3}{2} k_{b} T_{e}\right)$

The electron heat capacity $C_{e}$ is equal to the change of energy $U$ with respect to the electron temperature $T_{e}$. That is,

$C_{e}=\frac{\partial U}{\partial T_{e}}=\frac{3}{2} k_{b} T_{e}$

The overall expression to compute the change of energy in the electron subsystem reads

$\frac{\partial U}{\partial t}+\nabla \cdot \vec{W}=S+L$

in which $S$ and $L$ denote the source and loss terms respectively, and the term $\vec{W}$ on the left hand side of the equation describes the diffusion of electronic energy. This diffusion is the result of carrier-carrier scattering, which is described by [16]

$$
\vec{W}=-k_{e} \nabla T_{e}
$$

and

$k_{e}=\frac{2 k_{b}^{2} T_{e} n_{e}\left(\mu_{e}+\mu_{h}\right)}{e}$

in which $k_{\mathrm{e}}$ is the electron thermal conductivity, $\mu_{e}$ is the electron mobility and $\mu_{h}$ is the hole mobility. Sapphire has a low hole mobility so that $\mu_{h}$ may be neglected [4, 22]. Substituting expression (2.14) into (2.16) will result in a differential equation

$$
C_{e} \frac{\partial T_{e}}{\partial t}+\nabla \cdot \vec{W}=S+L-\frac{\partial n_{e}}{\partial t}\left(E_{g}+\frac{3}{2} k_{b} T_{e} ;\right.
$$

On the right hand side of equation (2.19) are source terms $S$, which describe an increase of energy due to multiphotonionization and free electron absorption, and loss terms $L$ that describe transfer of energy from the electron subsystem to the lattice (via the two temperature model, see below).

Multiphoton ionization will excite electrons with an excess kinetic energy and will therefore add energy to the free electron subsystem. The energy rate per volume unit can be determined by multiplying the ionization rate $W_{m p i}$ by the energy of the absorbed photons [18, 34]
$S_{m p i}=W_{m p i} \hbar \omega n_{p h}$

in which $n_{p h}$ is the number of photons and $\hbar$ is the reduced Planck constant. Free electrons will gain extra kinetic energy when they absorb photons. This process will result in an increase of energy in the free electron subsystem. Free electron absorption can be described by a source term including a free electron cross section

$S_{a b}=\sigma_{a b} n_{e} I$

in which $\sigma_{a b}$ is the free electron cross-section. This type of source term is referred to as Coulomb heating. Karras et al. [35] determined the free electron cross-section for a 800 $\mathrm{nm}$ laser source to equal $(12.5 \pm 0.2) \cdot 10^{-18} \mathrm{~cm}$.

The main loss to the electron energy is due to the interaction of the electron subsystem with the lattice caused by electron-phonon scattering, described by the well-known Two Temperature Model (TTM) [16]. The TTM is derived under the assumption that electron and phonon energy transport is described by the classical Fourier law. This approach is valid as long as the time scale of changes of the temperature (field) is much larger than relaxation time of the free electrons [36]. The TTM describes a balance between the electron temperature $T_{e}$ and the lattice temperature $T_{l}$ and reads

$W_{T T M}=\Gamma\left(T_{e}-T_{l}\right)=\frac{C_{e}}{\tau_{r}}\left(T_{e}-T_{l}\right)$

in which the electron-phonon coupling coefficient $\Gamma$ may be expressed as $C_{e} / \tau_{r}$. Here $\tau_{r}$ is the characteristic electron-lattice relaxation time. The latter is chosen here to equal $\tau_{\mathrm{r}}=1$ ps [22].

Substituting source terms (equations (2.20) and (2.21)) and loss terms (equation (2.22)) in equation (2.19), results in the PDE describing the free electron temperature

$$
\begin{aligned}
C_{e} \frac{\partial T_{e}}{\partial t}+\nabla \cdot \vec{W}=S_{m p i} & +S_{a b}- \\
& W_{T T M}-\frac{\partial n_{e}}{\partial t}\left(E_{g}+\frac{3}{2} k_{b} T_{e}\right)
\end{aligned}
$$

\subsection{Lattice temperature}

The classical Fourier law describes the spatial and temporal lattice temperature $T_{l}$, which is the result of a source term $Q$ and heat conduction, and reads [37]:

$\rho C_{l} \frac{\partial T_{l}}{\partial t}+\nabla \cdot \overrightarrow{q_{l}}=Q$

in which heat conduction is described by $\overrightarrow{q_{l}}=-k_{l} \nabla T_{l}$ where $k_{l}$ denotes the thermal conductivity of the lattice. The source term $Q$ is the result of electron-phonon coupling (two temperature model, see previous subsection):

$\rho C_{l} \frac{\partial T_{l}}{\partial t}+\nabla \cdot \overrightarrow{q_{l}}=S_{T T M}$ 


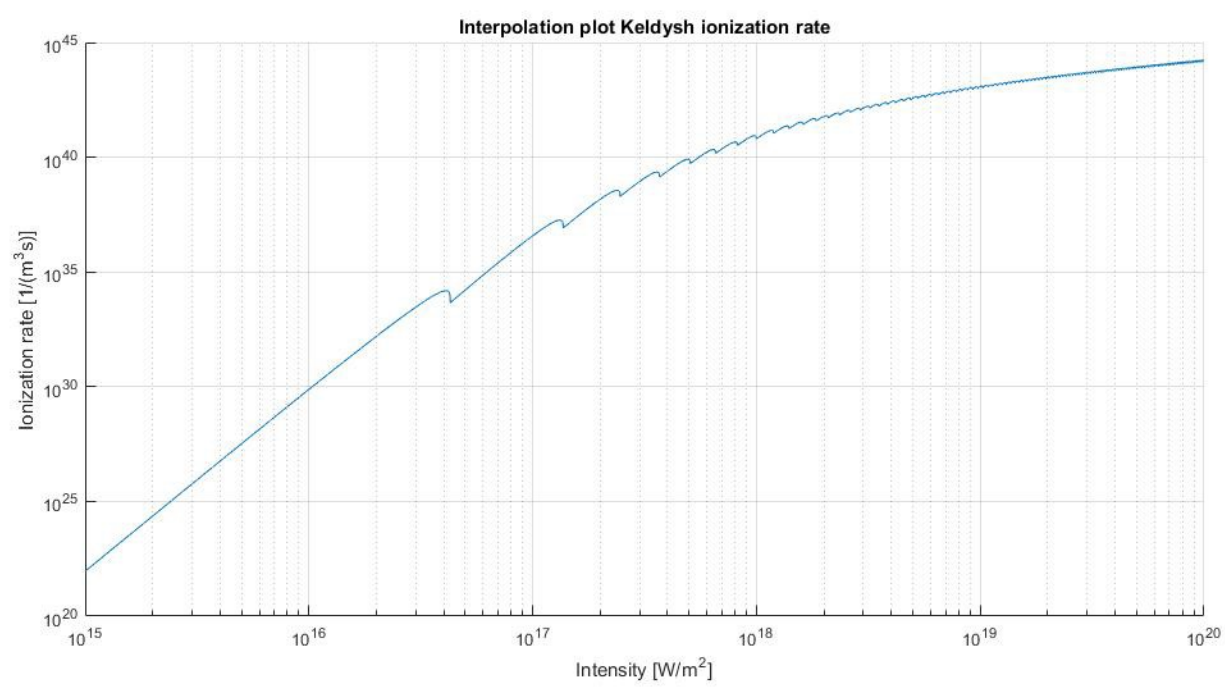

Fig. 2 Graphical representation of the lookup table of the Keyldysh ionization rate as function of the laser intensity.

\subsection{Beam propagation}

The mathematical complexity of describing both beam propagation and focussing proved to be difficult in the implementation of the model in COMSOL (see section 3). Therefore, the beam envelope is simplified to a collimatedi.e. a non-converging and non-diverging beam passing through the complete sapphire substrate. The laser intensity absorption can then be described by a beam optics approach.

Assuming laser beam propagation in the positive $z$-direction, a non-diverging beam and a volume absorbing material, the propagation losses can be described as [16]

$\frac{\partial I}{\partial z}=-S_{m p i}-S_{a b+}$

in which $S_{m p i}$ denotes the intensity loss of the laser beam due to multiphoton ionization. Note that the term $S_{m p i}$ is the same as the source term for the electron subsystem in equation (2.20). Intensity losses due to multiphoton ionization will be significant when multiphoton ionization rate is relevant. That is when the electron densities are low and when the Keldysh parameter $\gamma>>1$ [24]. The term $S_{a b}$ describes the intensity loss due to absorption of photons by conduction band electrons.

\section{Model implementation}

The PDEs were solved using the numerical software tool COMSOL Multiphysics ${ }^{\circledR}$ (COMSOL Inc.). An axis-symmetric simplified configuration on a time-dependent study was implemented.

It proved that the evaluating the Keldysh ionization rate, containing the complete elliptic integrals of the first and second kind (see section 2.1), was computationally too demanding. That is because the ionization rates $W_{p i}$, (equation (2.7)) and $W_{m p i}$ (equation (2.2)), need to be evaluated at every node on the calculation grid at every time-step. Therefore, the Keldysh ionization rates were implemented as a lookup table, which, via an interpolation, allows COMSOL Multiphys- ics ${ }^{\circledR}$ to compute an approximation of the full Keldysh ionization rate, as well as allow to approximate multiphoton absorption as function of the intensity. To that end, the

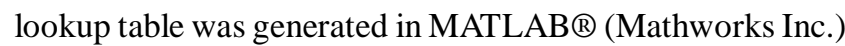
for a laser intensity range of $I=0$ to $10^{20}\left[\mathrm{~W} / \mathrm{m}^{2}\right]$ on a total of $10^{6}$ data points. Figure 2 shows this lookup table graphically.

\subsection{Initial and boundary conditions}

The beam diameter in the simulations was set to $w_{0}=50.15$ $\mathrm{nm}$ in order to reduce the computer memory requirements and computation time. This simplification can be considered reasonable for the model because only the absorption phenomena are simulated, without taking into account optical effects (i.e. diffraction).

Also, the pulse duration was set to $t_{p}=3 \mathrm{ps}$, for the same reason. The model would still be valid for a larger pulse duration (in the picosecond regime), but the effects of the resultant increase of electron density would need further investigation. The anisotropy of sapphire is neglected. At the first time instance, the electron and lattice temperatures are at equilibrium and were set to $300 \mathrm{~K}$. For both the lattice and electron temperature, all the boundary conditions, except for the symmetry axis, are set to a zero flux boundary conditions, as the thermal flux passing through the boundaries, at the time-ranges considered, will be negligible.

In the lattice the specific heat is assumed to be constant. At the first time instance, the electron density is set to equal to the density corresponding to thermally excited electrons at $300 \mathrm{~K}$. Applying these initial conditions to calculate the initial electron density results in a density of $3.18 \cdot 10^{-48} \mathrm{~m}^{-3}$. This low value is typical for large bandgap semiconductors at low temperatures. However, due to numerical restrictions, an initial electron density of eps $\approx 2.22 \cdot 10^{-16} \mathrm{~m}^{-3}$ was applied, which corresponds to the smallest number allowable due to machine precision. This value is much smaller than the multiphoton ionization rate occurring (just) before avalanche and tunnelling ionization will dominate. Hence, this value will not "trigger" avalanche ionization or significant free 
electron absorption. Therefore, the simulation results are not affected by setting the initial electron density to eps $\approx$ $2.22 \cdot 10^{-16} \mathrm{~m}^{-3}$ instead of $3.18 \cdot 10^{-48} \mathrm{~m}^{-3}$.

\section{Results}

This section presents the simulation results for various pulse energies listed in table 4 . The rounded values that will be used to refer to the simulations in this section are listed in the first column of the table. The simulations only converged for pulse energies which induce electron densities lower than the critical electron density, that is $n_{e}<n_{c r}$. That is, simulations where avalanche ionization was triggered resulted in an over critical electron density (plasma), see last column of table 4. The high absorption of the critical electron density resulted in extreme absorption rates in a very small (skin) layer of the material. The latter imposed numerical problems (failure to converge). Nevertheless, up to this instance the simulations provide interpretable results. Therefore, these results are included and discussed in the following subsections.

Table 4 Pulse energies considered for the simulations and maximum intensity reached

\begin{tabular}{ccc}
\hline $\begin{array}{c}\text { Pulse energy } \\
\text { simulation }\end{array}$ & Maximum intensity & $\begin{array}{c}\text { Critical electron } \\
\text { density reached? }\end{array}$ \\
\hline $0.06 \mu \mathrm{J}$ & $1.982 \cdot 10^{16} \mathrm{~W} / \mathrm{m}^{2}$ & No \\
$0.08 \mu \mathrm{J}$ & $2.972 \cdot 10^{16} \mathrm{~W} / \mathrm{m}^{2}$ & No \\
$0.11 \mu \mathrm{J}$ & $3.963 \cdot 10^{16} \mathrm{~W} / \mathrm{m}^{2}$ & No \\
$0.28 \mu \mathrm{J}$ & $9.908 \cdot 10^{16} \mathrm{~W} / \mathrm{m}^{2}$ & Yes \\
$0.45 \mu \mathrm{J}$ & $1.406 \cdot 10^{17} \mathrm{~W} / \mathrm{m}^{2}$ & Yes \\
$0.55 \mu \mathrm{J}$ & $1.515 \cdot 10^{17} \mathrm{~W} / \mathrm{m}^{2}$ & Yes \\
\hline
\end{tabular}

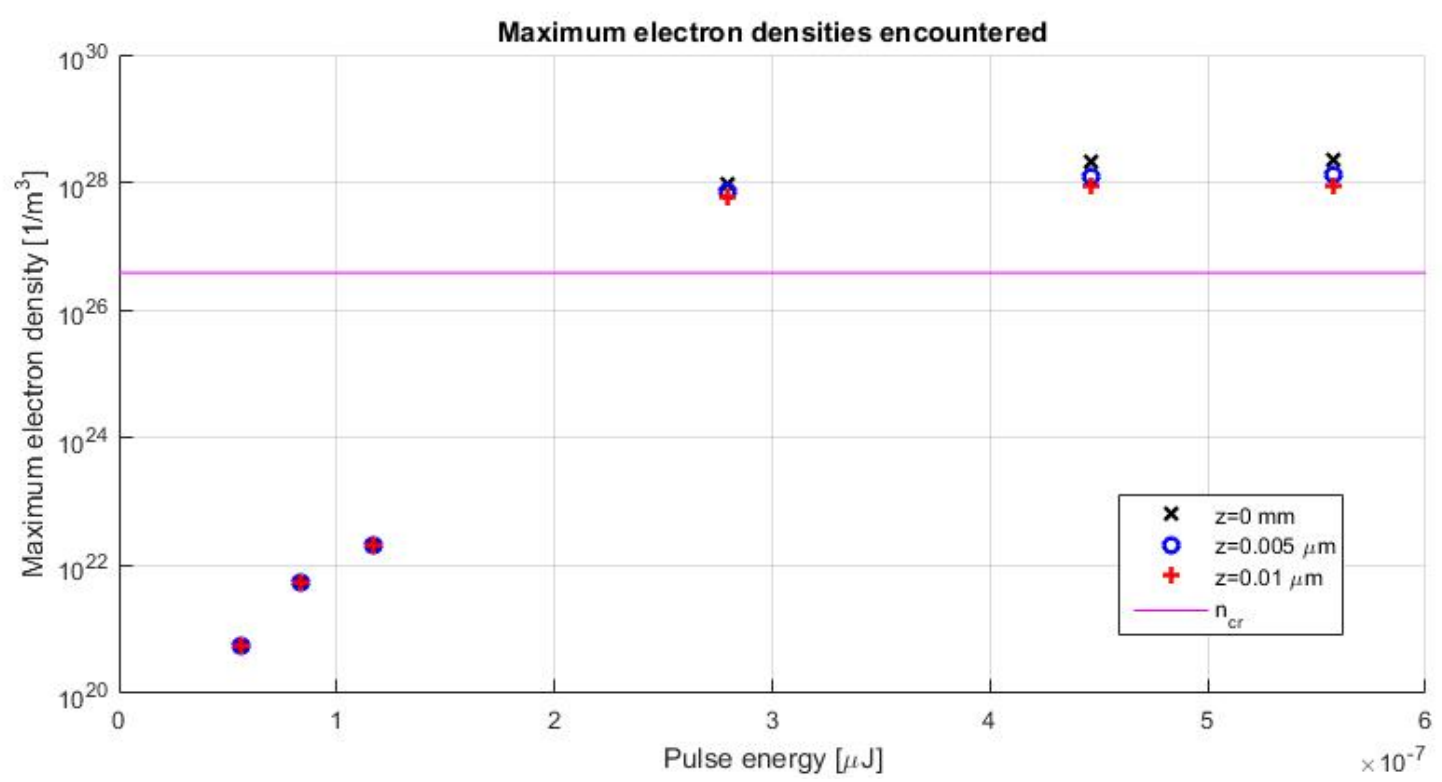

Fig. 3 Maximum electron densities $n_{\mathrm{e}}$ as function of the pulse energy at three different depths in the sapphire sample. The horizontal line indicates the critical electron density $n_{c r}=3.83 \cdot 10^{26}\left[\mathrm{~m}^{-3}\right]$.

\subsection{Electron density}

Figure 3 shows the maximum electron densities in the sample, as function of time for several pulse energies at three different locations: at the surface of the sample, at $5 \mathrm{~nm}$ and $10 \mathrm{~nm}$ below the surface of the sample. For low pulse energies, the maximum electron densities at different depths are close. That is, the data points in the graph coincide. This indicates that the absorption of photons in the material is small and the laser intensity, and electron densities do not vary with depth. From the graph it is clear that pulse energies over about $2.75 \mu \mathrm{J}$ induce a maximum electron density, which is over the critical electron density. It was found that, these simulations failed to converge as soon as the electron density reaches a value $n_{\mathrm{e}}>1.5 \cdot 10^{28} \mathrm{~m}^{-3}$. In addition, in the graph, the values of the maximum electron density for the corresponding pulse energies do not coincide. This indicates that the electron density at the locations near the surface prevents/reduces the ionization (rate) at locations further below the surface. 

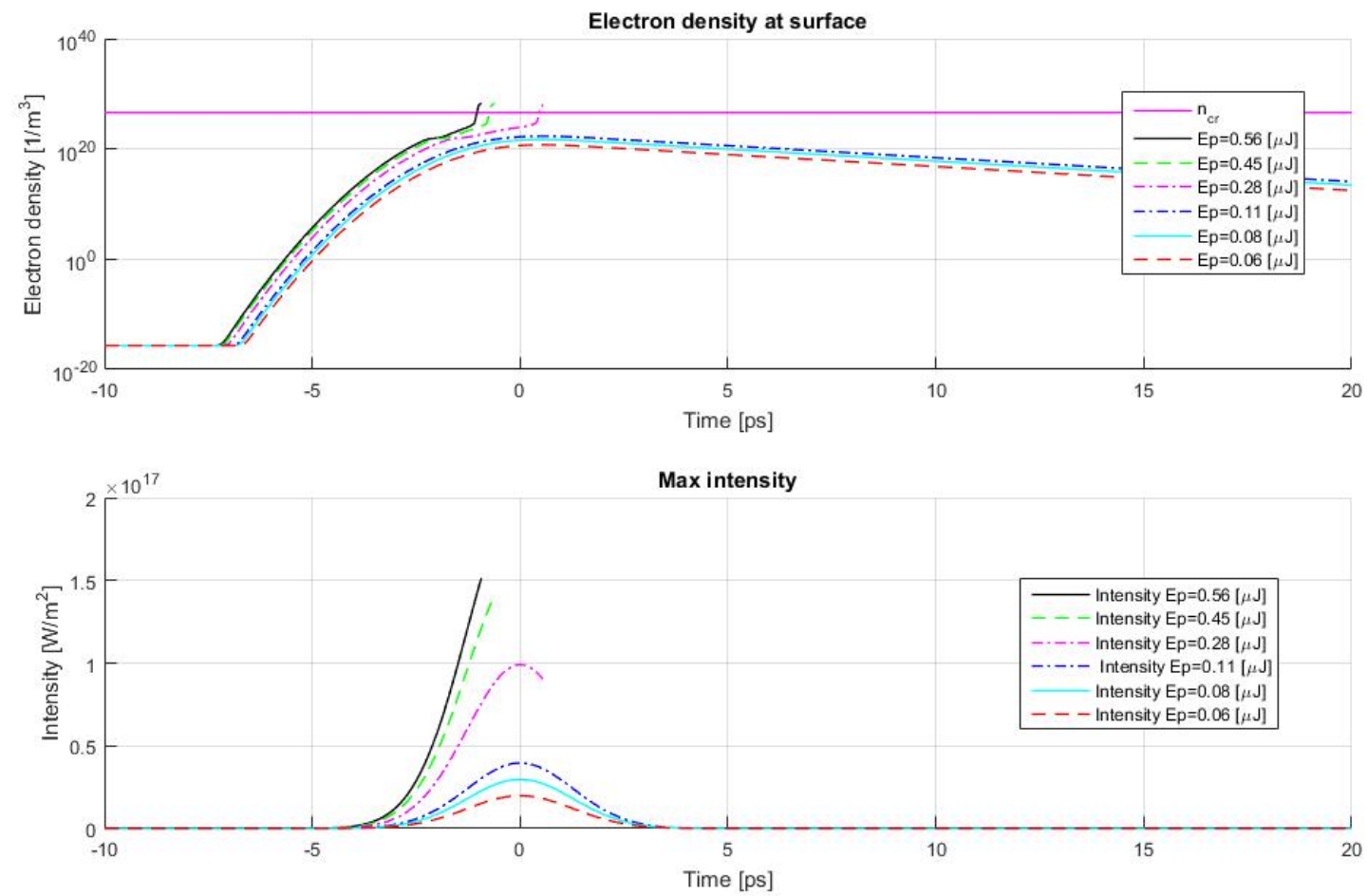

Fig. 4 Top: Maximum electron density as function of time at three different locations in the sample, for several pulse energies. The horizontal curve indicates the critical electron density $n_{\mathrm{r}}$. Bottom: Maximum laser intensity at the surface of the sample as a function of time, for several pulse energies. The time instance 0 ps represents the maximum intensity of the pulse (Gaussian in time). Negative and positive time steps in the graphs are relative to this time instance.
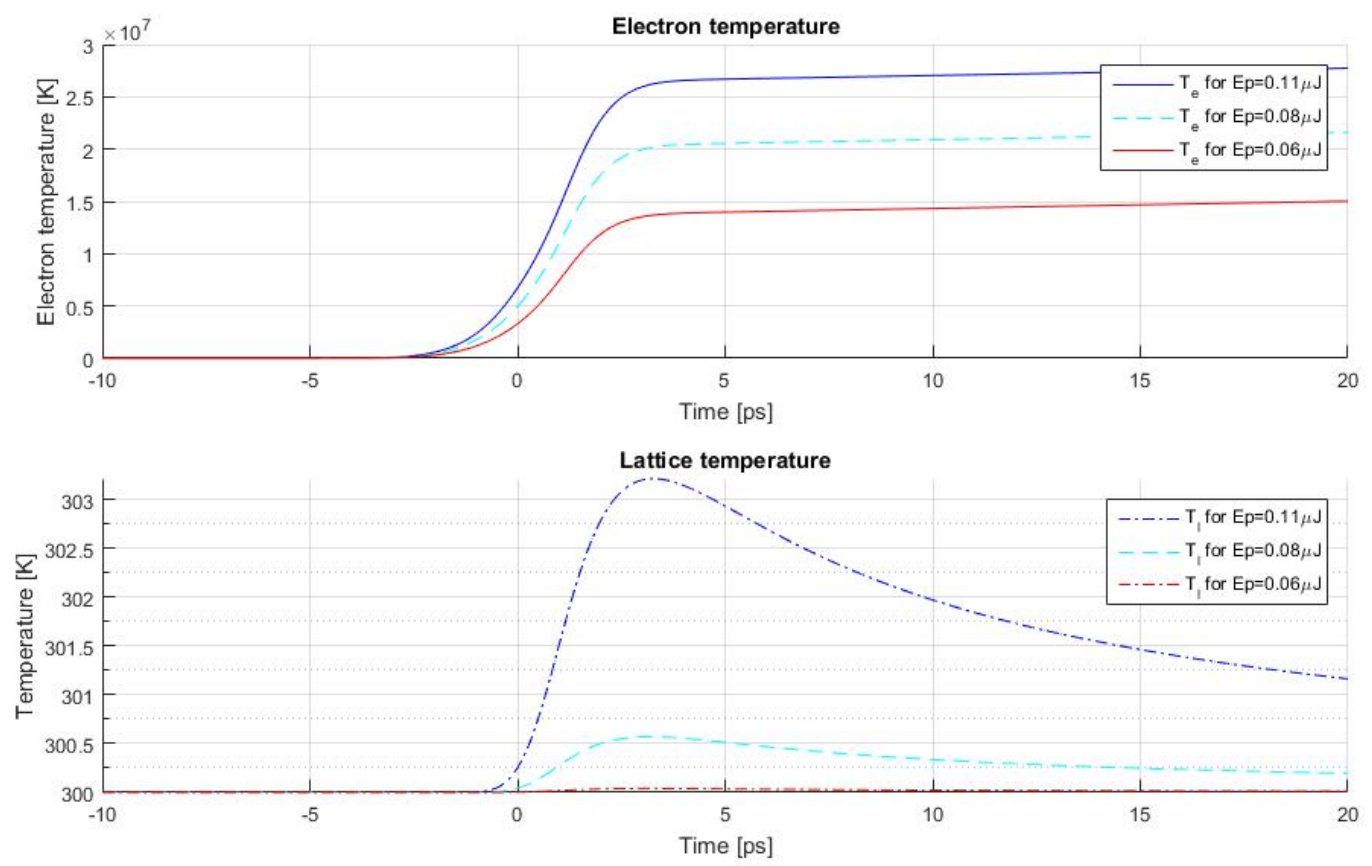

Fig. 5 Electron temperature $T_{\mathrm{e}}$ (top) and lattice temperature $T_{1}$ (bottom), as function of time, corresponding pulse energy levels leading to sub-critical electron densities ( $\left.n_{\mathrm{e}}<n_{\mathrm{cr}}\right)$. The time instance 0 ps represents the maximum intensity of the pulse (Gaussian in time). Negative and positive time steps in the graphs are relative to this time instance. 

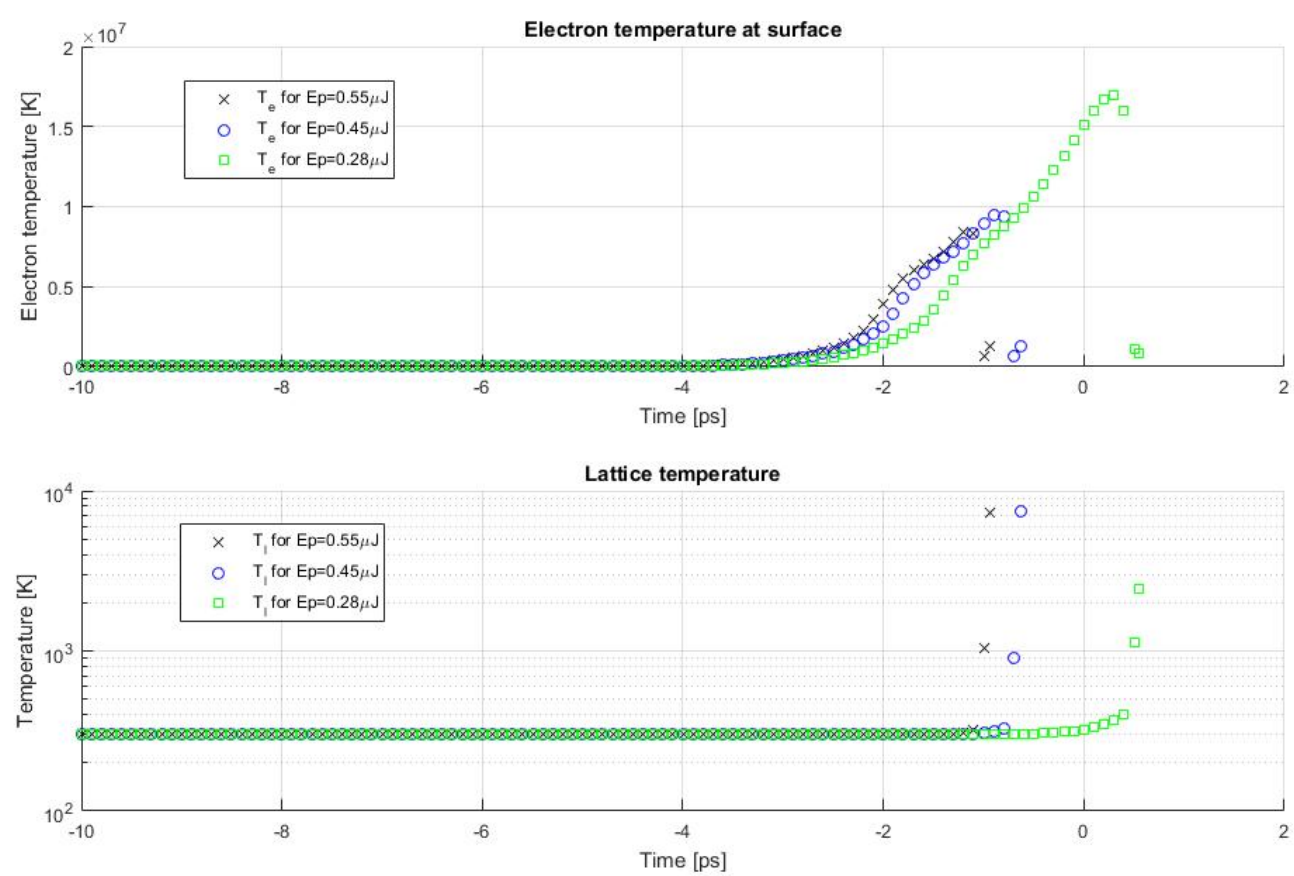

Fig. 6 Temperature plots critical $\left(n_{e}>n_{c r}\right)$ studies for corresponding pulse energies. (Top): electron temperature Te and (bottom): the lattice temperature $T l$. The time instance 0 ps represents the maximum intensity of the pulse (Gaussian in time). Negative and positive time steps in the graphs are relative to this time instance.

Figure 4 (top) shows the electron density as a function of time. Here it is shown that for low pulse energies, both the generation of free electrons and the decrease of electrons (due to recombination of electron-hole pairs) is non-linear but "smooth" in time. For high electron densities, that is $n_{e} \approx 5 \cdot 10^{23} \mathrm{~m}^{-3}$, the absorption and generation of free carriers by means of avalanche ionization results in a sudden increase in the electron density at the sample surface. In the graphs, this occurs at $\mathrm{t} \approx-2 \mathrm{ps}$ (where the time instance $0 \mathrm{ps}$ represents the maximum intensity of a Gaussian shaped laser pulse in time).

\subsection{Electron and lattice temperatures}

Figure 5 shows the electron and lattice temperatures in the material as function of time at several pulse energies. Here it is shown that the electron temperature increases until the pulse power has dropped significantly after 3 ps, see figure 5 (top). From this time instance on, the steep increase of the electron temperature does level off but still increases over time. This is due to the recombination of electron-hole pairs. Also, the lattice temperature induced by a pulse energy of $E_{\mathrm{p}}=0.11 \mu \mathrm{J}$ is much higher than the lattice temperature due to the pulse energy below $0.11 \mu \mathrm{J}$, see Figure 5 (bottom). This illustrates the non-linear increase in absorption rate due to multiphoton absorption. However, the difference in electron temperatures for the same pulse energy is much smaller (see figure 5 (top)), due to the fact that the energy in the electron subsystem depends on the electron temperature and specific heat (which depends on the electron density), where in the lattice the specific heat is assumed constant.

Figure 6 shows the electron and lattice temperatures as a function of time for pulse energies $E_{\mathrm{p}}=0.28 \mu \mathrm{J}, 0.45 \mu \mathrm{J}$ and $0.55 \mu \mathrm{J}$. At these pulse energies, electron densities over the critical electron densities were found. In these cases, the electron temperature shows a sudden decline at $t \approx-0.8 \mathrm{ps}$ (where the time instance 0 ps represents the maximum intensity of a Gaussian laser pulse in time). At this instance, avalanche ionization becomes significant, resulting in a temporary decrease in the electron temperature. This behavior was also observed by van Driel [21].

\subsection{Laser intensity spatial distribution}

At a pulse energy of $E_{\mathrm{p}}=0.11 \mu \mathrm{J}$ the absorption of photons in the material is negligible as the electron density is not sufficient to cause significant free electron absorption. As a result, the laser intensity distribution is not "distorted", but maintains its collimated "shape". Figure 7 (top) shows the laser intensity distribution for $E_{\mathrm{p}}=0.45 \mu \mathrm{J}$. From this graph, the effect of electron shielding can be observed, especially in the middle and right graph.

\subsection{Electron density distribution}

The electron density induced by a pulse energy of $E_{\mathrm{p}}=$ $0.11 \mu \mathrm{J}$ was found to be nearly constant along the z-axis. This is another indication that the electron density is too low to cause any significant free electron absorption.

At a higher pulse energy of $E_{\mathrm{p}}=0.45 \mu \mathrm{J}$, the electron densities are plotted in figure 7 (bottom) at three different time instances. Here the rapid increase in electron density due to the avalanche ionization can be observed, especially at $t=-0.6 \mathrm{ps}$. This graph also shows that the electron density is high near the surface of the sample.

Here, the locally high concentration of electrons implies an increased absorption rate and avalanche ionization rate, 


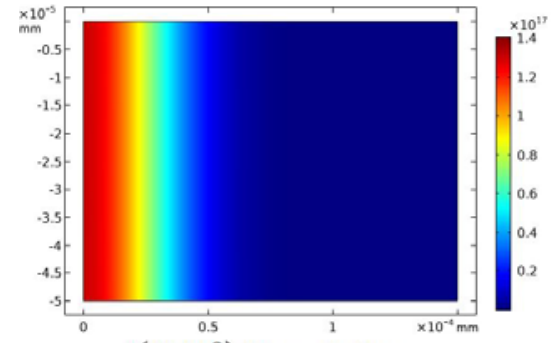

$\mathrm{I}\left(W / m^{2}\right) @ t=-0.8 \mathrm{ps}$

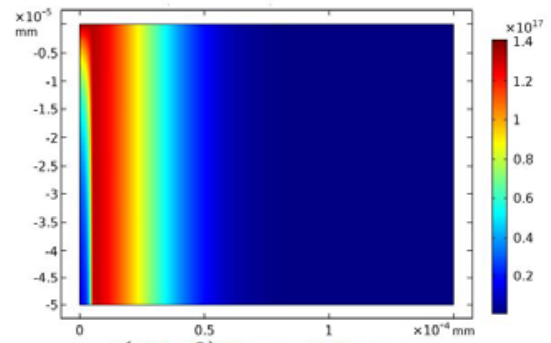

$\mathrm{I}\left(W / m^{2}\right) @ t=-0.7 p s$

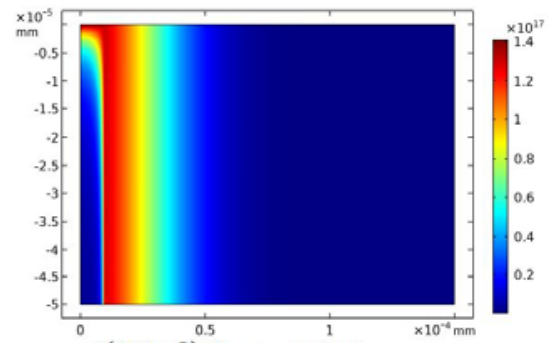

$\mathrm{I}\left(W / m^{2}\right) @ t=-0.6 p s$

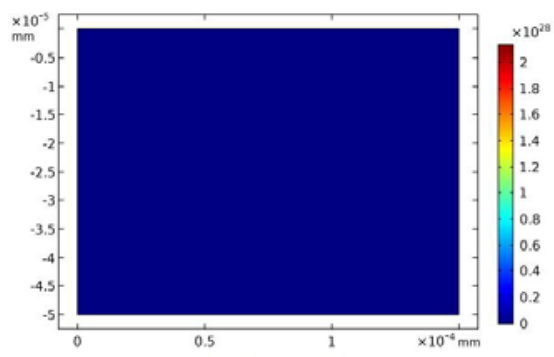

$n_{e}\left(m^{-3}\right) @ t=-0.8 \mathrm{ps}$

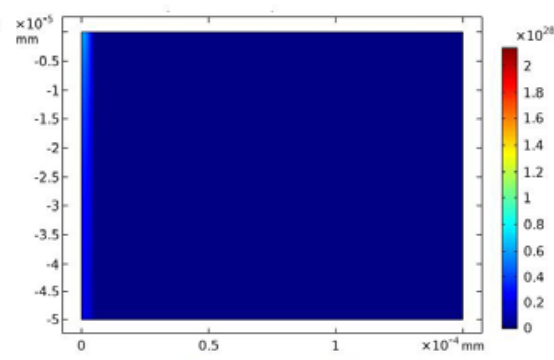

$n_{e}\left(m^{-3}\right) @ t=-0.7 p s$

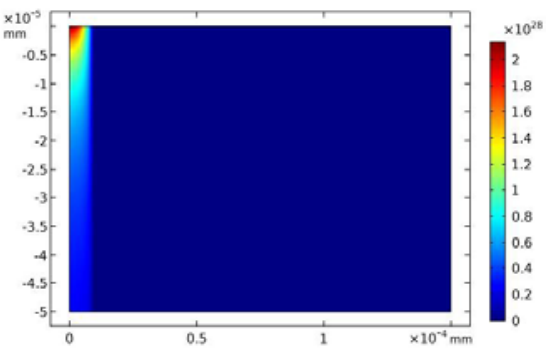

$n_{e}\left(m^{-3}\right) @ t=-0.6 p s$

Fig. 7 Top: Laser beam intensity distribution $I$ at several time instances induced by a pulse energy of $E_{p}=0.45 \mu \mathrm{J}$. Bottom: Electron density distributions $n_{e}$ for a pulse energy of $E p=0.45 \mu \mathrm{J}$. The distributions are axisymmetric and the graphs only show a 2D projection. The full cylindrical profiles shape can be imagined as a complete rotation $\left(360^{\circ}\right)$ of the shown distributions around the vertical axis. The vertical axis represents the optical axis where the laser beam is considered to propagate from top to bottom. The horizontal axis represents the

lateral distance from the optical axis.

which will result in an even higher electron density near the surface. The strong absorption lead to high spatial gradients in the laser intensity and electron density, which are hard to represent on a discrete mesh. This mechanism causes the convergence error that was encountered during the simulations with a critical electron density (see section 4.1).

\subsection{Temperature and energy distribution in the elec- tron subsystem}

Figure 8 shows the lattice temperature $T_{l}$ (left graphs) and the electron energy density $E_{e}$ (right graphs) for a pulse energy of $E_{\mathrm{p}}=0.11 \mu \mathrm{J}$. At $t=-3 \mathrm{ps}$, the graphs do not show any significant increase in $T_{1}$ nor $E_{\mathrm{e}}$. The highest electron energy density is reached at $t=1.2 \mathrm{ps}$. In contrast, the maximum lattice temperature is reached at $t=2.5 \mathrm{ps}$. The time difference between these maxima confirms the need for applying the two-temperature model. At $t=6 \mathrm{ps}$, the electron energy density has reduced significantly and some thermal conduction is present in the lattice. At the end of the simulation time $(t=20 \mathrm{ps})$ the electronic energy (density) is negligible. Also, the effects of heat conduction in the lattice become significant, as the spatial gradients are reduced and the energy has been spread over a larger volume in the sample.

\section{Limitations of the model}

\subsection{Phase transformation and material properties}

In the model, the material properties were assumed to be constant. In reality the conductivity and heat capacity of the material depend on the phase of the material, as well as on temperature [1]. Also, the anisotropy of sapphire was neglected, as the differences are small and it allows the application of an axis-symmetric simplification. The properties would in particular change when the material undergoes a phase transformation. This will affect the temperature distributions and should therefore be included when studying the lattice temperature distribution. The anisotropy may lead to a loss of axial symmetry of distributions, but the impact on the resulting is unknown and requires therefore more research.

A small change in the value for effective electron mass will significantly change the ionization rates, as stated by Jing [24]. The values reported have a range $\left(m^{*}{ }_{e}=0.35-\right.$ 0.38 ) which may cause a deviation from the actual ionization rates.

\subsection{Beam propagation and focusing optics}

The laser beam propagation in the governing equations was simplified to a collimated beam resulting in a system that is representative for surface processing. This rigorous simplification allows to demonstrate the phenomena during laser-material interaction of sapphire, but more work is required to include the beam propagation into the model in order to study subsurface laser-material interaction. Also, the beam propagation was taken one directional where in reality reflection at sample boundaries and at high electron densities may occur [7].

\subsection{Free electron temperature}

In section 2, it was shown that the electron temperature remains constant, after excitation, due to the recombination of electron-hole pairs and only converged to the lattice temperature when recombination ends. The same behavior was encountered in the simulations in the previous section, where the electron temperature keeps raising after the pulse maximum is reached, due to the recombination. 


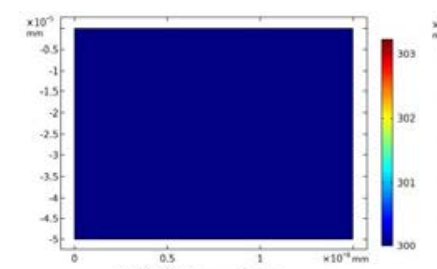

$T_{l}(K) @ t=-3 \mathrm{ps}$

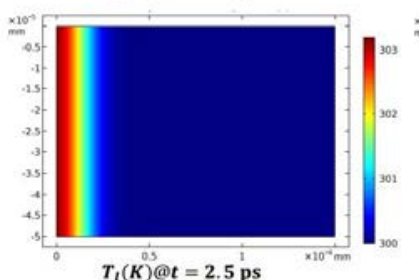

$T_{l}(K) @ t=2.5 \mathrm{ps}$

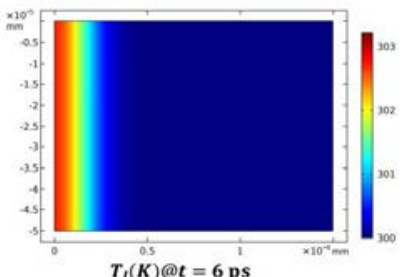

$T_{l}(K) @ t=6 \mathrm{ps}$

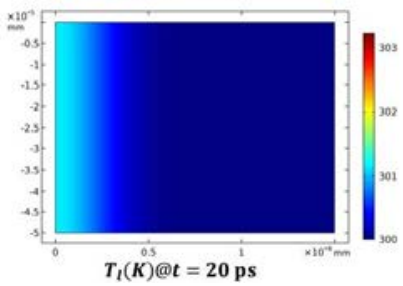

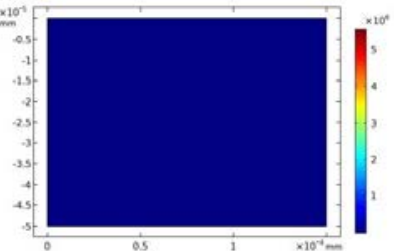

$E_{e}\left(J / m^{3}\right) @ t=-3 p s$

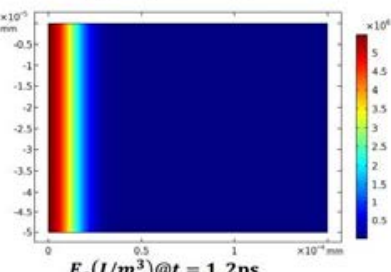

$E_{e}\left(J / m^{3}\right) @ t=1.2 p s$

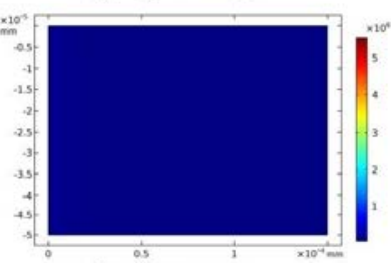

$E_{e}\left(J / m^{3}\right) @ t=-6 \mathrm{ps}$

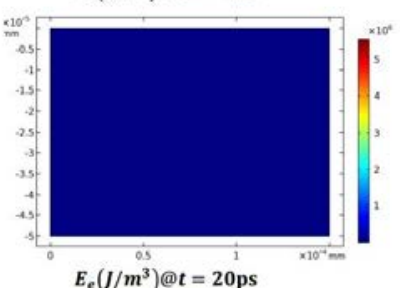

Fig. 8 (Left): lattice temperature distribution and (right) the electronic energy density at various time instances for a pulse energy of Ep $=0.11 \mu \mathrm{J}$. The distributions are axisymmetric and the graphs only show a 2D projection. The full cylindrical profiles shape can be imagined as a complete rotation $\left(360^{\circ}\right)$ of the shown distributions around the vertical axis. The vertical axis represents the optical axis where the laser beam is considered to propagate from top to bottom. The horizontal axis represents the lateral distance from the optical axis.

However, this behavior seems to be plausible, confirmed by [39] in which is shown that this occurs due to the recombination of electron-hole pairs that alone result in a temperature difference [38].

\subsection{Self-focussing and Kerr effect}

The simulation does not take into account self-focusing caused by the Kerr effect because this effect is not trigged under the laser conditions considered. That is, the critical power to trigger the Kerr effect depends on the nonlinear refractive index of sapphire ( $n 2)$ and is given by [39]

$P_{\text {crit }}=\frac{\lambda_{0}^{2}}{2 \pi n_{0} n_{2}}$

For the parameters studied in this paper, the critical power equals about 3.2 MW. This is one order of magnitude larger than the maximum peak power used in our study. Therefore, self-focusing is not modelled.

\section{Conclusions}

The objective of the study was to set the base for a numerical model that allows the simulation of the laser-material interaction in sapphire. Based on literature, a set of governing equations was established, describing the free electron density, the free electron temperature, the lattice temperature and beam propagation. The resulting model includes beam propagation of a collimated laser beam. The model was implemented and simulated in COMSOL Multiphysics ${ }^{\circledR}$. It was shown that the resulting model is capable of simulating the laser-material interaction during short ps pulse durations for sub-critical electron densities. The simulation results, when the critical electron density of $n_{c r}=3.83 \cdot 10^{26}\left[\mathrm{~m}^{-3}\right]$ was exceeded, failed to converge due to numerical issues. Nevertheless, the behavior of the model up to this density was as expected when compared to literature. Considering the lack in the literature of models describing jointly all the mentioned physical phenomena, this study can be seen as a significant contribution to a numerical model that is able to estimate both the absorption and the resulting thermal effects of the laser-material interaction in sapphire.

\section{Acknowledgements}

The Laser4Fun project (www.laser4fun.eu) leading to this study has received funding from the European Union's Horizon 2020 research and innovation programme under the Marie Skłodowska-Curie grant agreement No. 675063.

\section{References}

[1] E. R. Dobrovinskaya, L. A. Lytvynov, and V. Pishchik: "Application of Sapphire," in Sapphire (Springer US, 2009), pp. 1-54.

[2] D. Wortmann, J. Gottmann, N. Brandt, and H. HornSolle: "Micro- and nanostructures inside sapphire by fs-laser irradiation and selective etching," in 2008 Conference on Quantum Electronics and Laser Science Conference on Lasers and Electro-Optics, CLEO/QELS (2008).

[3] M. Hörstmann-Jungemann, J. Gottmann, and M. Keggenhoff: J. Laser Micro Nanoeng., 5, (2010) 145.

[4] J. Olivier and R. Poirier: Surf. Sci., 105, (1981) 347.

[5] S. Juodkazis and H. Misawa: SPIE Newsroom, (2007) 4.

[6] S. Juodkazis, K. Nishimura, H. Misawa, T. Ebisui, R. Waki, S. Matsuo, and T. Okada: Adv. Mater., 18, (2006) 1361.

[7] B. C. Stuart, M. D. Feit, S. Herman, A. M. Rubenchik, B. W. Shore, and M. D. Perry: Phys. Rev. B, 53, (1996) 1749.

[8] A.-C. Tien, S. Backus, H. Kapteyn, M. Murnane, and G. Mourou: Phys. Rev. Lett., (1999).

[9] K. Sokolowski-Tinten and D. von der Linde: Phys. Rev. B - Condens. Matter Mater. Phys., (2000).

[10] B. Rethfeld, A. Kaiser, M. Vicanek, and G. Simon: Phys. Rev. B, 65, (2002) 214303.

[11] J. R. Peñano, P. Sprangle, B. Hafizi, W. Manheimer, and A. Zigler: Phys. Rev. E. Stat. Nonlin. Soft Matter Phys., 72, (2005) 036412.

[12] E. Arola: IEEE J. Quantum Electron., 50, (2014) 1.

[13] N. M. Bulgakova: J. Laser Micro/Nanoengineering, 2, (2007) 76 . 
[14] N. M. Bulgakova, V. P. Zhukov, Y. P. Meshcheryakov, L. Gemini, J. Brajer, D. Rostohar, and T. Mocek: J. Opt. Soc. Am. B, (2014).

[15] K. I. Popov, C. McElcheran, K. Briggs, S. Mack, and L. Ramunno: Opt. Express, (2011).

[16] P. C. Verburg, G. R. B. E. Römer, and A. J. Huis In 'T Veld: Appl. Phys. A Mater. Sci. Process., 114, (2014) 1135.

[17] T.-L. Chang, Z.-C. Chen, and Y.-C. Lee: Opt. Express, 20, (2012) 15997.

[18] P. C. Verburg, G. R. B. E. Römer, and A. J. Huis In 'T Veld: Appl. Phys. A Mater. Sci. Process., (2014).

[19] S. K. Sundaram and E. Mazur: Nat. Mater., 1, (2002) 217.

[20] L. V. Keldysh: Sov. Phys. JETP, 20, (1965) 1307.

[21] H. M. Van Driel: Phys. Rev. B, 35, (1987) 8166.

[22] N. M. Bulgakova, R. Stoian, A. Rosenfeld, and D.Berlin: 5448, (2004) 121.

[23] B. Van Zeghbroeck.: "Principles of semiconductor devices.," .

[24] X. Jing, Y. Tian, J. Zhang, S. Chen, Y. Jin, J. Shao, and Z. Fan: Appl. Surf. Sci., 258, (2012) 4741.

[25] K. Starke, D. Ristau, H. Welling, T. V. Amotchkina, M. Trubetskov, A. A. Tikhonravov, and A. S. Chirkin: 5273, (2004) 501.

[26] F. Wang, J. Shan, E. Knoesel, M. Bonn, and T. F. Heinz: "Electronic charge transport in sapphire studied by optical-pump/THz-probe spectroscopy," in Ultrafast Phenomena in Semiconductors and Nanostructure Materials VIII, K.-T. Tsen, J.-J. Song, and H. Jiang, eds. (2004), Vol. 5352, p. 216.

[27] T. Q. Jia, H. X. Chen, M. Huang, F. L. Zhao, X. X. Li, S. Z. Xu, H. Y. Sun, D. H. Feng, C. B. Li, X. F. Wang, R. X. Li, Z. Z. Xu, X. K. He, and H. Kuroda: Phys. Rev. B - Condens. Matter Mater. Phys., 73, (2006) 1.

[28] X. Jing, Y. Tian, J. Zhang, S. Chen, Y. Jin, J. Shao, and Z. Fan: Appl. Surf. Sci., 258, (2012) 4741.

[29] N. M. Bulgakova, R. Stoian, A. Rosenfeld, I. V. Hertel, and E. E. B. Campbell: Phys. Rev. B Condens. Matter Mater. Phys., 69, (2004) 1.

[30] R. Stoian, A. Rosenfeld, D. Ashkenasi, I. V. Hertel, N. M. Bulgakova, and E. E. Campbell: Phys. Rev. Lett., 88, (2002) 976031.

[31] N. M. Bulgakova, V. P. Zhukov, and Y. P. Meshcheryakov: Appl. Phys. B Lasers Opt., 113, (2013) 437.

[32] M. Lundstrom: Meas. Sci. Technol., 13, (2002) 230.

[33] J. Shan, F. Wang, E. Knoesel, M. Bonn, and T. F. Heinz: Phys. Rev. Lett., 90, (2003) 4.

[34] E. Gamaly, B. Luther-Davies, A. Rode, S. Joudkazis, H. Misawa, L. Hallo, P. Nicolai, and V. Tikhonchuk: J. Phys. Conf. Ser., (2007).

[35] C. Karras, Z. Sun, D. N. Nguyen, L. A. Emmert, and W. Rudolph: 8190, (2011) 819028.

[36] B. R. and D. S. I. and M. E. G. and S. I. Anisimov: J. Phys. D. Appl. Phys., 50, (2017) 193001.

[37] Y. A. Çengel and A. J. Ghajar: Heat and Mass Transfer A Practical Approach (2014).

[38] T. L. F. Leung and H. M. Van Driel: Appl. Phys. Lett., 45, (1984) 683.
[39] E. G. Gamaly, S. Juodkazis, K. Nishimura, H. Misawa, and B. Luther Davies: Phys. Rev. B, 73, (2006) 214101.

(Received: June 26, 2018, Accepted: October 7, 2018) 